Chapter 19

\title{
ERAS (Enhanced Recovery after Surgery) in Colorectal Surgery
}

\author{
Raúl Sánchez-Jiménez, Alberto Blanco Álvarez, \\ Jacobo Trebol López, Antonio Sánchez Jiménez, \\ Fernando Gutiérrez Conde and \\ José Antonio Carmona Sáez
}

Additional information is available at the end of the chapter

http://dx.doi.org/10.5772/57136

\section{Introduction}

Evidence-based medicine has led to an extensive investigation and development of new therapies and programs to improve the care of the surgical patient, both in the postoperative and in the pre-operative period, known as enhanced recovery after surgery (ERAS) programs, "fast-track" programs or multimodal rehabilitation programs.

\subsection{Definition}

ERAS programs are evidenced-based protocols designed to standardize and optimize perioperative medical care in order to reduce surgical trauma, perioperative physiological stress and organ dysfunction related to elective procedures [1]. In addition, improved outcomes, decreased hospital length of stay and faster patient recovery to normal life are expected to be obtained. Other advantages of this philosophy are the reduction of clinical complications and the health costs together with and increase of patient satisfaction. A diagram with all the core principles of an ERAS program can be seen on Figure 1.

This approach could not be understood and implemented without the participation and commitment of a multidisciplinary team including surgeons, anesthesiologists, nursing staff and hospital administration. Moreover, it is important to make the patient and their families a partner in their care and give them join responsibility for the recovery.

These kinds of programs are not exclusive of a type of surgery or surgical procedure since they can be applied to different specialties (digestive, vascular, thoracic, etc.), different procedures 
(colon resection, pancreatic procedures, etc.) or different approaches (laparoscopic or open procedures).

In this chapter we will focus on ERAS protocols applied to colorectal surgery.

\subsection{Background}

Patients undergoing major open colorectal surgery traditionally undergo prolonged rehabilitation and complication rates even as high as 30\% have been reported after this procedure [2].

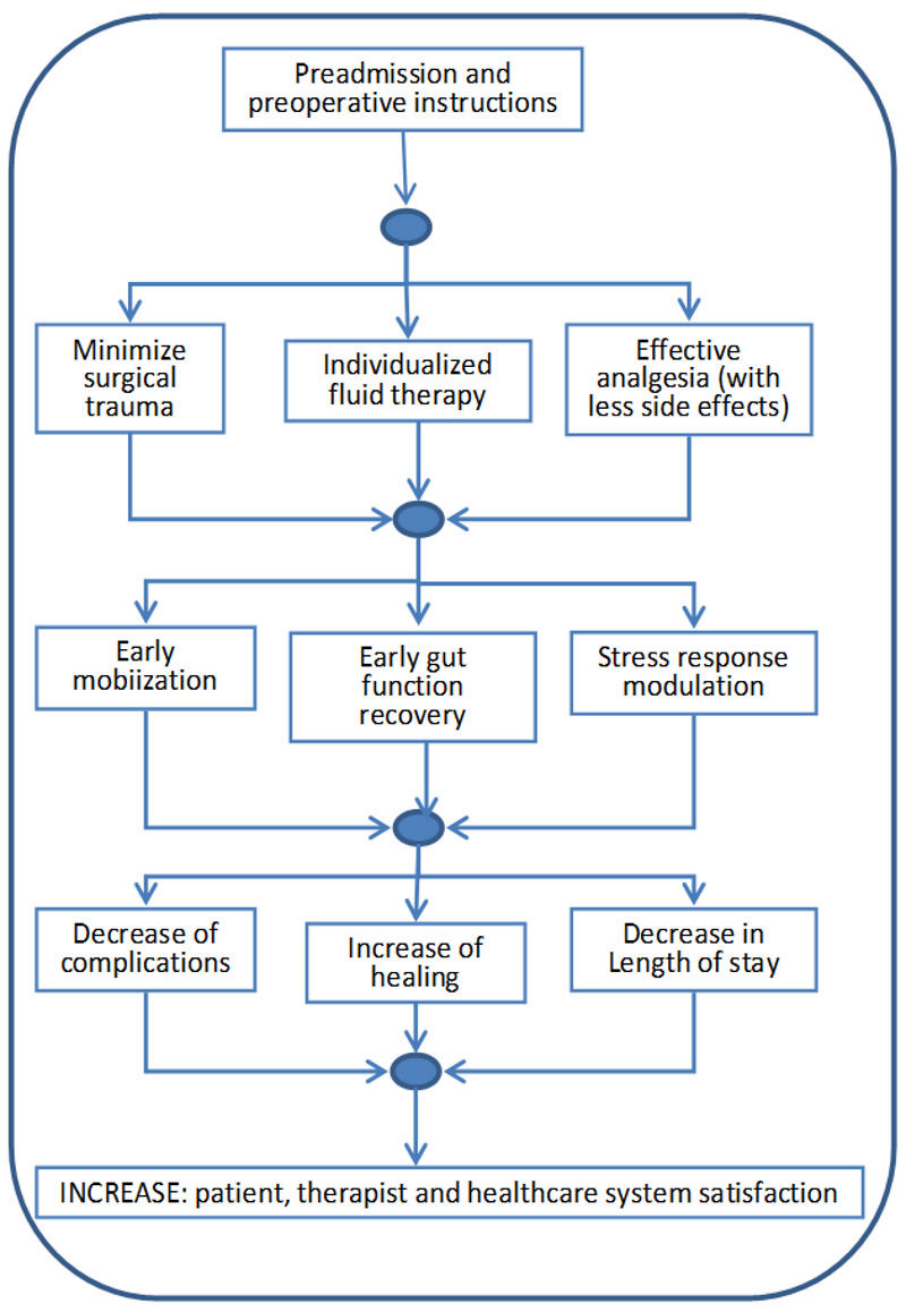

Figure 1. Core principles of an ERAS program applied to digestive tract surgery. 
Surgeons learned over the years that surgery was an aggression and that the bigger the procedure was, the bigger the aggression emerged. For example, surgeons understood that patients undergoing major open colorectal surgery suffered prolonged rehabilitation with profound changes in endocrine, metabolic, neural and pulmonary function during the postoperative period. However, the scientific interest was not focused on how to control these changes.

In digestive surgery there were some inviolable principles that were transferred between generation of surgeons over a long period of time. Senior clinicians had strong principles and they were assumed as a dogma. We will highlight some of them:

- Preoperative prolonged fasting is necessary to empty the bowel, prevent intraoperative contamination and the early passage of bowel content through an anastomosis.

- Mechanical bowel preparation is imperative in colorectal surgery to prevent intraoperative contamination and the passage of faeces through a suture line while it is healing. This passage could increase leaking and dehiscence risk or infections.

- Systematic use of nasogastric tubes is imperative to empty stomach and prevent its content to come into the bowel protecting sutures.

- Drains usage is essential in all kind of digestive procedures.

- Extended periods of bed rest are recommended to facilitate abdominal wall healing.

- Postoperative period is a "resting time" in which surgeons are expecting spontaneous patient recovery.

The majority of these paradigms were only based on clinical experience instead of the scientific evidence and, subsequently, they were passed down from masters to disciples, who preserved them as a non-questionable tradition. However, stepwise, published studies have dispelled these and other "truths" and the evidence has taught us that some of them may be unnecessary and maybe they can contribute to postoperative functional deterioration. For example, the return of bowel function is essential for postoperative recovery and this is influenced negatively by several perioperative factors such as preoperative fasting and bowel mechanical preparation, opioid analgesic, fluid overload, immobilization and postoperative prolonged fasting. Thus, several reviews and meta-analyses have focused in the absence of benefits in routinely mechanical bowel preparation, routine nasogastric decompression or prolonged postoperative fasting [3].

In 1990's, several revolutionary changes were seen: in the field of anesthesia the development of regional anesthetic techniques and new drugs to control pain and sedation; and in the field of surgery the widespread use of minimally invasive (laparoscopic) techniques. As a result, a great improvement in postoperative recovery and earlier return of patients to normal function were achieved. Moreover, it was thought that a minimally invasive approach, with reduced operative trauma, conducted to an earlier return of bowel function and allowed for early oral tolerance. The next step was the thinking that some of the improvements seen were simply due to overall changes in perioperative care attitudes. 
In the late 1990's, based on those findings, the "fast track" concept to major abdominal surgery was pioneered by Professor Henrik Kehlet and a solid doctrine concerning perioperative care was born. He was a researcher surgeon interested in perioperative medicine, from the Hvidovre University Hospital in Denmark. Kehlet and colleages were investigating in combined pain relief, early feeding and mobilization since 1995 [4], observing that no more complications were seen and that patients even could be discharged earlier [3]. The concept of a "multimodal" approach was first published in 1997 [4] and subsequently prospective studies appeared [5]. The aims of Kehlet's study were to reduce postoperative morbidity and mortality and to promote a faster recovery through a multimodal approach, thus minimizing the impact of the factors that lead to surgical stress. On the other hand, in the study of Basse et al the multimodal rehabilitation program significantly reduced the postoperative hospital stay in high-risk patients undergoing colonic resection (two days compared to more than 10 days in some historical series) and it might also reduce postoperative ileus and cardiopulmonary complications [5].

During the following decade published studies in this issue grew exponentially. Subsequently, cohort studies, controlled trials and several reviews and meta-analyses were published. It is important to highlight those from Wind [6], Goubas [7], and the meta-analyses directed by Cochrane Collaborative Group in 2011 that will be analyzed in the following chapter's sections [8]. Moreover, an ERAS Society was officially founded in 2010 as a natural evolution of the ERAS Study Group. This group started its works in 2001 trying to change from tradition to best-practice because there was a great discrepancy between the existing practices and those which were already known to be best practice based on the existing literature. More information is provided in the official website http://www.erassociety.org/.

To summarize, we can conclude that published results and their meta-analyses have shown the benefits of this package of measures, so that evidence-based medicine supports the ERAS concept. Nevertheless, recent surveys have demonstrated slow adaptation and implementation of the fast-track methodology. In this setting, it has been shown by Kehlet et al in an international multicenter study based on 1,082 patients who had undergone elective colonic operations that strategies that could contribute to improved recovery and reduce complications were not been applied and that major improvements in outcomes and reduction of costs could be obtained applying ERAS methodology [9].

Little by little, ERAS implementation and application in the clinical setting continued growing in the following years until the present. Nowadays ERAS protocols, with little modifications to adapt them to each center's functioning, are been applied in a great number of colorectal units worldwide. The information communicated in different conventions and published makes us think that ERAS has changed from a promising "published" issue to a real application in the clinical practice.

\subsection{The stress response to surgery}

Surgeons have shown interest in metabolic and endocrine response to the surgical trauma long time ago. Such interest has increased by the recognition that to modulate this response to the surgical aggression might reduce the postoperative morbidity and mortality. 
The overall metabolic changes in the stress response involve protein and fat cabolism to provide energy. Protein from skeletal muscle and glycerol from fat breakdown are utilised in glucogenogenesis in the liver. In addition surgery induces hormonal, haematological and inmunological changes and activate the sympathetic nervous system (stimulated by hypotension, hypoxaemia or metabolic acidosis, pain, anxiety and distress, autonomic and afferent nerves and directly hypothalamus) [10]. The initial stimulus for this response comes from cytokines, especially IL-6 and TNF, release by leucocytes and endotelial cells present at the site of injury and they are the principal mediators of the response in the acute-phase. Postoperative levels of these cytokines are correlated with the magnitude of the surgery and the presence of complications. On the other hand, leucocytes are key effector cells in the response to surgery, they mobilize quickly to devitalizated or injured tissue to begin repair and prevent secondary microbial invasion. A few minutes after the start of surgery an ACTH, vasopresine, cortisol, catecholamines, aldosterone and glucagon release occur pretending to provide to the disabled organism energy, to retain liquid and salt, and supporting the cardiovascular homeostasis [11].

A randomized controlled trial has shown that Multimodal Rehabilitation programs attenuate the response to the surgical stress as it demonstrates a significant descent of IL-1, IL-6, TNF$\alpha$ and INF-gamma levels in the postoperative period.

Summarizing, the stress response to surgery increase the levels of ACTH, cortisol, GH, IGF1, $\mathrm{ADH}$ and glucagon, reduce the insulin, mobilizes glycogen (by glycogenolysis and skeletal muscle breakdown) and promotes formation of acute phase proteins and lipolysis.

This response also generates adverse effects; some of the most important are:

- Increased myocardial oxygen demand.

- Hypoxaemia.

- Splanchnic vasoconstriction wich may impact intestinal anastomoses healing.

- Exhaustion of energy supplies and loss of lean muscle mass, leading to weakness of both peripheral and respiratory muscle if it is severe.

- Impaired wound healing and increased risk of infections.

- Hypercoagulability (risk of Deep Vein Thrombosis).

- Sodium and water retention.

The response to the surgical trauma is protective since his final target is the survival of the disabled organism. It depends on a delicate balance between pro-inflammatory and antiinflammatory mechanisms; nevertheless, it is known that it can be harmful when this balance is altered. Thus, if the pro-inflammatory component predominates, a Systemic Inflammatory Response Syndrome (SIRS) could be induced; on the other hand patients can suffer the effects derived from the inmunosupresion as infections or tumor progression if predominates antiinflammatory components. 


\section{Aim and concerns}

The aims of ERAS programs are:

- To standardize and optimize perioperative medical care.

- To atenuate the stress response to surgery: metabolic, endocrine and inflamatory response as well as reduce protein cathabolism.

- To decrease hospital length stay and a faster patient recovery to normal life.

- Regarding hospital discharge, factors such as pain, lack of gastrointestinal function and immobility complications are the main delaying patient discharge after colorectal surgery. So ERAS objectives will be to promote pain control, to improve gastrointestinal function and to avoid immobility.

- Despite the discharge criteria with ERAS programs are similar than in traditional care, patients usually reach these criteria sooner.

\section{ERAS protocol components}

ERAS programs are composed of preoperative, intra-operative and postoperative strategies combined to form a multimodal pathway:

\subsection{Preoperative}

\subsubsection{Pre-admission}

Pre-operative optimization: it is focused on targeting areas to optimize patient comorbidities (previous or related to the presenting complaint) such as anemia, diabetic and blood pressure control, optimizing cardiovascular disease treatments, respiratory functioning,... It is also imperative avoid smoking and alcohol consumption. Patient's individualized Risk stratification is also important to make good patient information and treatment decision.

Information: It is shown that this information reduces the patient's anxiety and facilitates the compliance of the program [12].

Patients and their families should be very knowledgeable about the process. It is very important to make them a partner in the process and give them the responsibility for their recovery and they should be clearly informated about the perioperative care, normal course of the protocol, discharge criteria, possible complications and the outpatient follow-up after discharge. Targets like postoperative oral intake or early mobilization are given in this stage to the patient.

Patient education: including ostomy management and its appropiate localization for it. 
Pre-operative nutritional management: drinks and any new medication and nutritional supplements should be given at this time.

\subsubsection{Pre-operative care}

Admission on the day of surgery: because the patient has been prepared for surgery in the preadmission period.

\section{Pre-operative fasting and carbohydrate loading:}

- Fasting is required to reduce the risk of aspiration during a general anesthesia The duration of preoperative fasting should be two hours for liquids and six hours for solids (grade A recommendation) [13].

- Major surgery is associated with postoperative insulin-resistance. Non-diabetic patients should receive carbohydrate $(\mathrm{CHO})$ loading pre-operatively because they increase glycerol deposits, reduce thirst, hunger and postoperative insulin resistance [14], reducing protein catabolism, postoperative ileus and loss of lean muscle mass. $\mathrm{CHO}$ has to be taken in the evening before surgery and 2 hours before anaesthetic induction [15].

\section{Avoid mechanical bowel preparation:}

Mechanical bowel preparation can cause dehydration and fluid and electrolyte abnormalities, particularly in elderly patients, increasing morbidity and post-operative ileus [16].

\section{Medication:}

- Medication causing long-term sedation from midnight prior to surgery must not been used, in order to conserve the sleep pattern (grade A recommendation).

- Prophylaxis against thromboembolism with low-dose unfraccionated heparin or lowmolecular-weight heparin (grade A recommendation) and the use of elastic stockings or pneumatic compression are recommended.

- Antibiotic prophylaxis with single-dose antibiotic prophylaxis against both anaerobes and aerobes about one hour before surgery is recommended (grade A recommendation).

\subsection{Intraoperative}

\section{Normothermia:}

Changes in body temperature can lead to coagulopathy, adverse cardiac events, and decreased resistance to surgical wound infections. An upper-body forced-air heating cover should be used routinely (grade A recommendation).

\section{Prevention of post-operative ileus:}

Mid-thoracic epidural analgesia and avoidance of fluid overload are recommended to prevent post-operative ileus (grade A recommendation) [16], [17]. 


\section{Approach:}

The use of minimally invasive techniques, where possible is advisable. Laparoscopic approach is recommended if locally validated (grade A recommendation) [18]. It has been shown to reduce the length of hospital stay, initial wound complications and time to return of gastrointestinal tract function in colorectal surgery. If an open procedure is required, transverse incisions should be made preferentially to reduce postoperative pain.

\section{Peri-operative fluid management:}

Perioperative fluid management for fast-track protocols must be balanced between avoiding hypovolemia and excessive fluid administration. Overhydration has previously been common in the perioperative period, and comparisons of liberal and restrictive fluid regimes suggest that this may be detrimental.

Perioperative fluid overload can cause fluid retention and increase body weight; this is related with generalized edema (which can cause a descense in tissue oxygenation [19]), visceral edema (related with postoperative ileus), can impaire wound and anastomosis healing, can increase cardiorespiratory complications $[20,21]$ and also thrombotic risk.

Intra-operative and post-operative fluid restriction in major colonic surgery with avoidance of hypovolaemia is safe (grade A recommendation) and reduce the time for return of gastrointestinal tract function, improves healing, reduce length of hospital admission and avoid pulmonary dysfunction [21] and reduce overall postoperative complications by up to two thirds [22]. Early commencement of oral intake also allows reducing intravenous fluids sooner. Postoperative serious hypotension may best treated with vasopressors rather than large quantities of intravenous fluids.

No clear consensus exists regarding the optimal fluid (crystalloid or colloid), the fluid amount (liberal, restricted or supplemental) and the fluid administration (goal-directed fluid therapy by oesophageal Doppler-derived variables -such as stroke volume, the blood volume pumped with each beat- versus conventional haemodynamic variables) for fluid management after and during colectomy.

Fluid management can be then optimized using transesophageal monitoring of the cardiac stroke volume with goal-directed administration of fluid boluses. This methodology can improve outcome (patients recovered gut function significantly faster and suffered significantly less gastrointestinal and overall morbidity) in patients with significant medical comorbidities allowing an earlier hospital discharge [23]. These results have been confirmed with posterior literature review that showed a reduced hospital stay, fewer complications and ICU admissions, less requirement for inotropes and faster return of normal gastro-intestinal function [24].

In the last years literature reviews and metaanalyses have been published trying to give light to these doubts: which fluid, how many and how to control the administration. We want to highlight the one from Rahbari et al [25]. Authors included nine randomized controlled trials, finding that restrictive fluid amount (OR 0.41 with $95 \%$ CI 0.22 to $0.77 ; \mathrm{P}=0.005)$ and goaldirected fluid therapy by means of oesophageal Doppler-derived variables (OR 0.43 with 955 
CI 0.26 to $0.71 ; \mathrm{P}=0.001$ ) significantly reduced overall morbidity after colorectal resection compared with standard fluid amount and fluid therapy guided by conventional haemodynamic variables respectively. No significant differences were founded in mortality, cardiopulmonary morbidity, wound infection, anastomotic failure, recovery of bowel function and hospital stay.

\section{Nasogastric tubes:}

They should be inserted only if ileus develops. They are associated with discomfort and a delay in oral intake. Nasogastric tubes should not be used routinely in the elective situations in postoperative period (grade A recommendation) [26],[27].

\section{Surgical drains:}

Drains are avoided, as there is no evidence of beneficial effect in reducing postoperative morbidity, mortality, or reduce the effect of anastomotic leakage [28],[29]. Short-term (24-hour) use of drains after low anterior resections may be advisable. They are not indicated following routine colonic resection above the peritoneal reflection.

\section{Epidural analgesia:}

The aim of their use is to reduce the dose of general anesthetic needed and the stress response to surgery. In order to reduce the release of stress hormones and post-operative insuline resistance it is very important start with the epidural analgesia before the surgery. (Grade A recommendation).

\subsection{Postoperative}

\section{Hydration:}

Maintenance of hydration, avoiding overcharge and encouraging the discontinuation of intravenous fluid therapy as soon as possible and early commencement of oral intake, including carbohydrate drinks.

\section{Analgesia:}

Patients should receive continuous epidural mid-thoracic low-dose local anesthetic and opioid combinations (grade A recommendation) for approximately 48 hours following elective colonic surgery and approximately 96 hours following pelvic surgery. This provides postoperative analgesia and reduces postoperative ileus by blockade of the sympathetic nervous system. Low concentration local anesthetic mixtures reduce motor block and improve early mobilization. Intravenous analgesia is used with paracetamol and non-esteroid anti-inflammatory drugs [30]. Intravenous opioids are avoided because of increase sedation, ileus and respiratory complications.

\section{Nausea and vomiting:}

It is very important a risk stratification of patients during surgery using the Apfel scoring system with prophylaxis given for moderate or high risk patients. Risk factors are: female sex, non-smokers, administration of opioids postoperatively, motion sickness or previous postop- 
erative nause and vomitig [31]. Patients with two ore more risk factors should be treated. Dexamethasone or 5HT3 receptor antagonist, droperidol or metoclopramide near the end of surgery are recomended. It is preferred those medication that have a minimal post-operative hang-over and effects on gastrointesinal motility. Also short-acting anesthetic and analgesic agents should be used, avoiding long-lasting opiates where possible [32].

\section{Nutrition support:}

Early commencement of an oral intake (frequently in theater recovery) after surgery should be encouraged (grade A recommendation). Oral nutritional supplements should be prescribed (approximately $200 \mathrm{~mL}$, energy dense, 2-3 times daily) from the day of surgery until normal food intake is achieved. These supplements can be continued beyond the return of normal intake if pre-operative nutritional status is poor. Early resumption of oral intake is associated with fewer wound infections and shorter hospital admissions as well.

\section{Early mobilization:}

Early mobilization should occur in accordance with pre-operative plan and is a key element of ERAS in colorectal surgery [10]. For patients to be out of bed for two hours on the day of surgery and six hours thereafter is recommended. The aim is to reduce muscle loss and improve respiratory function, reducing the risk of pneumonia, and maximizing oxygen delivery to tissues. This is also essential to reducing the risk of venous thromboembolism. The breathing exercises should be done, especially in patients with previous lung pathology and these exercises must be trained before surgery.

\section{Urinary catheter and drains:}

Urinary catheters and peritoneal drains should bre removed as soon as possible in order to reduce the incidence of urinary tract infection and because of early mobilization respectively

\section{Early discharge:}

At the end, early discharge, when the discharge criteria have been reached, is the goal of fasttrack along with the early recovery and return to normal activity.

A summary of all of these commented components of the perioperative management can be seen on Figure 2.

\section{From theory to practice - How to organize an ERAS program}

- A well-educated multidisciplinary team will be needed composed by: surgeons, anesthesiologists and pain care specialists, nursing staff, physiotherapysts and occupational therapists and social workers

- ERAS programs involve a selected number of individual interventions. It is necessary to implement all together, because only in this way they demonstrate a greater impact on outcomes than when we implement them as individual interventions [1],[33]. 


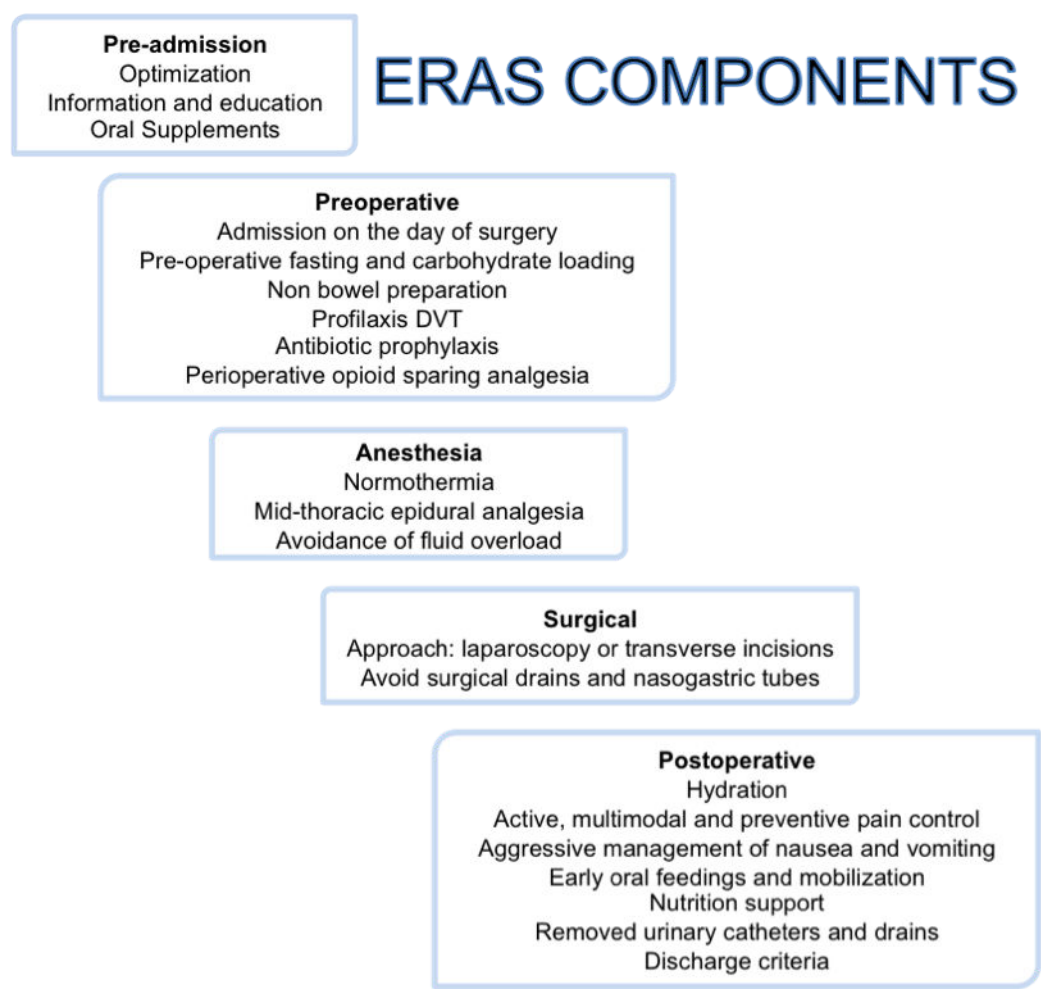

Figure 2. Components of the ERAS protocols

- It is necessary a review of the literature and a carefully study of the hospital resources where the ERAS program will be implemented.

- The program should be designed in agreement with consensus documents.

- A systematic audit should be performed including length of stay, morbidity, mortality and hospital readmissions to allow direct comparison with other institutions and provide motivation for staff and patients.

An example of an ERAS protocol in colorectal surgery can be seen on Table 1.

\section{PREOPERATIVE}

\begin{tabular}{ll}
\hline Pre-admission & Indication for surgery, information and signed consent \\
& Optimization of the patient and education (including stoma) \\
& Preoperative studies (anesthetist and other if is required) \\
Hyperproteic supplement 3 times every day during the week before surgery
\end{tabular}




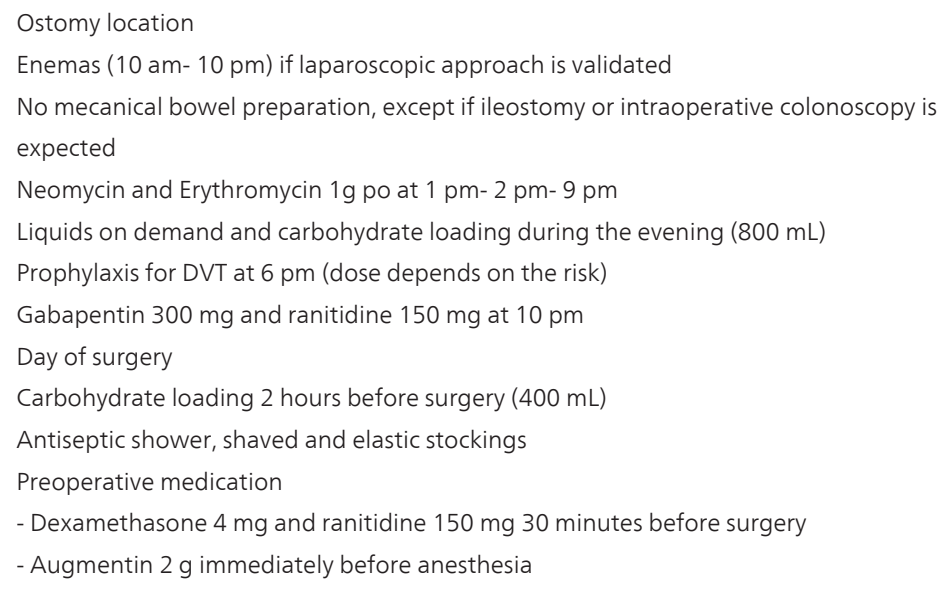

\section{INTRAOPERATIVE}

Anesthetist Normothermia: upper-body forced-air heating cover and liquid heater $\left(37^{\circ} \mathrm{C}\right)$ Mid-thoracic (T10) epidural analgesia with levobupivacaine

Maintenance Oxygen/air FiO2 0.6 - 0.8

Avoid fluids overload maintaining $5 \mathrm{cc} / \mathrm{kg} / \mathrm{h}$ (Hartmann). $\mathrm{Hb}>8.0 \mathrm{~g} / \mathrm{dL}$

Vasoconstrictive drugs if hypotension

Ondansetron $4 \mathrm{mg}$ or droperidol $1.25 \mathrm{mg} 30$ minutes before the end

Adittional dose of Augmentine $2 \mathrm{~g}$ if surgery takes more than $4 \mathrm{~h}$

Remove nasogastric tube at the end of surgery

\begin{tabular}{ll}
\hline Surgeons & Laparoscopy or transverse incisions \\
& Avoid surgical drains except short-term (24-hour) drains after low anterior resection \\
& Local anesthesia with levobupivacaine
\end{tabular}

\section{POSTOPERATIVE}

Day of surgery Mask with $4 \mathrm{l} / \mathrm{m}$ oxygen flow for $2 \mathrm{~h}$ independent of saturation, after that nasal cannulae for (Recovery room) $\quad$ SpO2 $>95 \%$

Epidural analgesia according with protocol of anesthesia

Day of surgery In seat for at least $2 \mathrm{~h}$ in the evening

Fluids: $1.5 \mathrm{~L}$ Ringer lactate solution +0.5 expander fluids $\times 24$ hours

Paracetamol $1 \mathrm{~g} / 6 \mathrm{~h}+/$ - metamizol $2 \mathrm{~g} / 8 \mathrm{~h}$

Epidural analgesia according to protocol of anesthesia

Metroclopramide $10 \mathrm{mg} / 8 \mathrm{~h}$ and ondansetron $4 \mathrm{mg} / 8 \mathrm{~h}$

Nasal cannulae for $\mathrm{SpO} 2>95 \%$

Liquid diet 2 hours after surgery including $400 \mathrm{~mL}$ of Hyperproteic supplement

Postoperative day $1 \quad$ Mobilization $6 \mathrm{~h}$ a day

Suspend fluid e.v. if tolerated diet, maintaining heparine injection

Paracetamol 1g/6h +/- metamizol 2g/8h

Epidural analgesia according to protocol of anesthesia 
Liquid diet at least $2 \mathrm{~L}$, including $600 \mathrm{~mL}$ of high protein/high calories

Laxative /12h with MgO (2g / 24h)

Heparine in order to protocol

Ranitidine t.d.s

\begin{tabular}{ll}
\hline Postoperative day 2 & See day 1 recommendations \\
& Mobilisation on demand \\
& Remove epidural catheter \\
& Bland/normal diet including $600 \mathrm{~mL}$ of high protein/high calorie \\
& Verify if Discharge criteria have been reached by the patient \\
\hline Postoperative day 3 & See day 2 recommendations \\
& Normal diet including $600 \mathrm{~mL}$ of high protein/high calorie \\
& Verify if Discharge criteria have been reached by the patiente \\
\hline Follow-up & Telephone monitoring for $48 \mathrm{~h}$ \\
& Out-patient visit after 10-14 daysRanitidine v.o. / $8 \mathrm{~h}$ \\
\hline
\end{tabular}

Table 1. An ERAS protocol example in colorectal resections.

\section{Discharge criteria}

The goal of ERAS programs is an accelerated recovery and return to normal activity but it is not the only focus of the protocol [34]. Discharge criteria and time-based discharge depends on the community support and possibility to follow-up.

Patients and their families should feel comfortable with the discharge. In this setting they should know that they will be followed as outpatient and they could return to hospital if required.

Discharge criteria must be previously established (see Table 2):

\section{Discharge criteria}

Good mobilization

Adequate oral intake for liquids and solids

Gastrointestinal transit for gas

Normal urinary function

No wound problems

Pain control

No fever

Patient know about possible complications and their detection

Patient feel comfortable with discharge

Table 2. Discharge criteria most usually used in colorectal surgery ERAS programs. 


\section{Outcomes}

The expanding evidence-based medicine shows that ERAS program benefits not only all patients (including the elderly or potentially malnourished patients) but also the health service [35].

Patients accomplish surgery in the best condition. They have better management during and after operation and the best post-operative recovery.

Randomized trials and meta-analysis identified a significantly shorter length of stay and lower in-hospital postoperative complications (maybe secondary to the shorter length of hospital stay) [6].These advantages are mainly attributed to fluid restriction and epidural analgesia.

Other outcome improvements attributed to ERAS programs are shorter duration of postoperative ileus [6], better oral intake, better pain control, less cardiopulmonary morbidity, better preservation of body mass and exercise performance [36], an improvement in grip strength (all of them suggesting an overall improvement in muscular function), earlier resumption of normal activities and a reduced need for daytime sleep [37].

Early discharge is the goal of Fast-Track protocols, and should not be offset by a higher rate of hospital readmission. However, the overall rate of readmission for patients managed with early discharge is comparable to patients with a longer median length of hospital stay [34].Regarding the economical issues, it must be pointed out that the increased cost in laparoscopic approach must be balanced with savings from a shorter length of hospital stay, lower morbidity and no differences in readmission rates.

\section{The research initiatives}

The confirmation of the initial results should prompt the ERAS methodology embracing in other kind of major surgical procedures as gastric or pancreatic procedures.

The possibility of applying some components of fast-track programs in patients undergoing emergency colorectal surgery must be also evaluated, especially in order to reduce preoperative stress.

New drugs like Ketamina, Lidocaina, Alvimopan could have an important role in the future because of their properties in analgesia and in gastrointestinal resumption.

\section{Summary and recommendations}

ERAS programs for colorectal surgery were developed to reduce inpatient hospital costs through improvements in preoperative, intra-operative and postoperative strategies. 
The success of this program depends on pre-operative setting of expectations including the concept of patients being partners in their care and taking part-ownership of post-operative rehabilitation.

Best results are achieved when the whole multidisciplinary team believe and take part in the program and individual interventions are implemented all together.

The keys of ERAS are: patient information, preservation of gastrointestinal function, minimize organ dysfunction, active pain control and to promote the patient's autonomy.

Early discharge is the goal of ERAS protocols and patients usually reach the discharge criteria sooner than in traditional care.

Although most of the studies tend to find a lower morbidity, there are no clear advantage in mortality and we think that more studies are needed to confirm the results and focalized in mortality and long-term results of ERAs methodology. We can conclude that at least there are no significant differences in mortality and morbidity with traditional care (ERAs methodology is not dangerous for patients and probably represents a big benefice) and ERAS are more costeffectiveness than traditional care.

\section{Author details}

Raúl Sánchez-Jiménez ${ }^{1 *}$, Alberto Blanco Álvarez², Jacobo Trebol López ${ }^{1}$, Antonio Sánchez Jiménez ${ }^{3}$, Fernando Gutiérrez Conde ${ }^{4}$ and José Antonio Carmona Sáez ${ }^{1}$

*Address all correspondence to: raulsj34@gmail.com

1 Department of General Surgery, Nuestra Señora de Sonsoles Hospital, Ávila, Spain

2 Department of General Surgery, Santos Reyes Hospital, Burgos, Spain

3 Physiotherapist, Cadiz University, Cádiz, Spain

4 Department of General Surgery, University Hospital of Salamanca, Salamanca, Spain

\section{References}

[1] Kehlet H, Wilmore DW. Fast-track surgery. Br.J.Surg. 2005;92:3-4.

[2] Kehlet H, Mogensen T. Hospital stay of 2 days after open sigmoidectomy with a multimodal rehabilitation programme. Br.J.Surg. 1999;86:227-230.

[3] Kehlet H. Multimodal approach to control postoperative pathophysiology and rehabilitation. Br.J.Anaesth. 1997;78:606-617. 
[4] Basse L, Hjort JD, Billesbolle P, Werner M, Kehlet H. A clinical pathway to accelerate recovery after colonic resection. Ann.Surg. 2000;232:51-57.

[5] Wind J, Polle SW, Fung Kon Jin PH et al. Systematic review of enhanced recovery programmes in colonic surgery. Br.J.Surg. 2006;93:800-809.

[6] Gouvas N, Tan E, Windsor A, Xynos E, Tekkis PP. Fast-track vs standard care in colorectal surgery: a meta-analysis update. Int.J.Colorectal Dis. 2009;24:1119-1131.

[7] Spanjersberg WR, Reurings J, Keus F, van Laarhoven CJ. Fast track surgery versus conventional recovery strategies for colorectal surgery. Cochrane.Database.Syst.Rev. $2011 C D 007635$.

[8] Kehlet H, Buchler MW, Beart RW, Jr., Billingham RP, Williamson R. Care after colonic operation--is it evidence-based? Results from a multinational survey in Europe and the United States. J.Am.Coll.Surg. 2006;202:45-54.

[9] Zutshi M, Delaney CP, Senagore AJ, Fazio VW. Shorter hospital stay associated with fastrack postoperative care pathways and laparoscopic intestinal resection are not associated with increased physical activity. Colorectal Dis. 2004;6:477-480.

[10] Desborough JP. The stress response to trauma and surgery. Br.J.Anaesth. 2000;85:109-117.

[11] Carli F, Charlebois P, Baldini G, Cachero O, Stein B. An integrated multidisciplinary approach to implementation of a fast-track program for laparoscopic colorectal surgery. Can.J.Anaesth. 2009;56:837-842.

[12] Practice guidelines for preoperative fasting and the use of pharmacologic agents to reduce the risk of pulmonary aspiration: application to healthy patients undergoing elective procedures: an updated report by the American Society of Anesthesiologists Committee on Standards and Practice Parameters. Anesthesiology 2011;114:495-511.

[13] Soop M, Carlson GL, Hopkinson J et al. Randomized clinical trial of the effects of immediate enteral nutrition on metabolic responses to major colorectal surgery in an enhanced recovery protocol. Br.J.Surg. 2004;91:1138-1145.

[14] Mathur S, Plank LD, McCall JL et al. Randomized controlled trial of preoperative oral carbohydrate treatment in major abdominal surgery. Br.J.Surg. 2010;97:485-494.

[15] Guenaga KK, Matos D, Wille-Jorgensen P. Mechanical bowel preparation for elective colorectal surgery. Cochrane.Database.Syst.Rev. 2009CD001544.

[16] Kehlet H. Postoperative ileus--an update on preventive techniques. Nat.Clin.Pract.Gastroenterol.Hepatol. 2008;5:552-558.

[17] Vlug MS, Wind J, Hollmann MW et al. Laparoscopy in combination with fast track multimodal management is the best perioperative strategy in patients undergoing colonic surgery: a randomized clinical trial (LAFA-study). Ann.Surg. 2011;254:868-875. 
[18] Lobo DN, Bostock KA, Neal KR et al. Effect of salt and water balance on recovery of gastrointestinal function after elective colonic resection: a randomised controlled trial. Lancet 2002;359:1812-1818.

[19] Cheatham ML, Chapman WC, Key SP, Sawyers JL. A meta-analysis of selective versus routine nasogastric decompression after elective laparotomy. Ann.Surg. 1995;221:469-476.

[20] Petrowsky H, Demartines N, Rousson V, Clavien PA. Evidence-based value of prophylactic drainage in gastrointestinal surgery: a systematic review and meta-analyses. Ann.Surg. 2004;240:1074-1084.

[21] Urbach DR, Kennedy ED, Cohen MM. Colon and rectal anastomoses do not require routine drainage: a systematic review and meta-analysis. Ann.Surg. 1999;229:174-180.

[22] Francom M. Stop drug price increases. Am.Pharm. 1991;NS31:6, 8.

[23] Tiippana EM, Hamunen K, Kontinen VK, Kalso E. Do surgical patients benefit from perioperative gabapentin/pregabalin? A systematic review of efficacy and safety. Anesth.Analg. 2007;104:1545-56, table.

[24] Kehlet H, Holte K. Review of postoperative ileus. Am.J.Surg. 2001;182:3S-10S.

[25] Leslie JB, Viscusi ER, Pergolizzi JV, Jr., Panchal SJ. Anesthetic Routines: The Anesthesiologist's Role in GI Recovery and Postoperative Ileus. Adv.Prev.Med. 2011;2011:976904.

[26] Wilmore DW, Kehlet H. Management of patients in fast track surgery. BMJ 2001;322:473-476.

[27] Hendren S, Morris AM, Zhang W, Dimick J. Early discharge and hospital readmission after colectomy for cancer. Dis.Colon Rectum 2011;54:1362-1367.

[28] Adamina M, Kehlet H, Tomlinson GA, Senagore AJ, Delaney CP. Enhanced recovery pathways optimize health outcomes and resource utilization: a meta-analysis of randomized controlled trials in colorectal surgery. Surgery 2011;149:830-840.

[29] Basse L, Madsen JL, Kehlet H. Normal gastrointestinal transit after colonic resection using epidural analgesia, enforced oral nutrition and laxative. Br.J.Surg. 2001;88:1498-1500.

[30] Jakobsen DH, Sonne E, Andreasen J, Kehlet H. Convalescence after colonic surgery with fast-track vs conventional care. Colorectal Dis. 2006;8:683-687. 
